\title{
OPEN Association of maternal history of neonatal death with subsequent neonatal death across 56 low- and middle-income countries
}

\author{
Zhihui Li ${ }^{1}$, Mudit Kapoor ${ }^{2}$, Rockli Kim ${ }^{3,4,5 \bowtie}$ \& S. V. Subramanian ${ }^{5,6 \bowtie ~}$
}

Early identification of high-risk pregnancies can reduce global neonatal mortality rate. Using the most recent Demographic and Health Surveys from 56 low- and middle-income countries, we examined the proportion of mothers with history of neonatal deaths. Logistic regression models were used to assess the association between maternal history of neonatal death and subsequent neonatal mortality. The adjusted models controlled for socioeconomic, child, and pregnancy-related factors. Country-specific analyses were performed to assess heterogeneity in this association across countries. Among the 437,049 live births included in the study, 6910 resulted in neonatal deaths. In general, $22.4 \%$ (1549) occurred to mothers with previous history of neonatal death; at the country-level, this proportion ranged from $1.2 \%$ (95\% confidence interval $[\mathrm{Cl}] 0.0,2.6)$ in Dominican Republic to $38.1 \%(95 \% \mathrm{Cl}$ 26.0, 50.1) in Niger. Maternal history of neonatal death was significantly associated with subsequent neonatal death in both the pooled and the subgroup analyses. In the fully adjusted model, history of neonatal death was associated with $2.1(95 \% \mathrm{Cl} 1.9,2.4)$ times higher odds of subsequent neonatal mortality in the pooled analysis. We observed large variation in the associations across countries ranging from fully adjusted odds ratio (FAOR) of $0.4(95 \% \mathrm{Cl} 0.0,4.0)$ in Dominican Republic to 16.1 $(95 \% \mathrm{Cl} 3.6,42.0)$ in South Africa. Our study suggests that maternal history of neonatal death could be an effective early identifier of high-risk pregnancies in resource-poor countries. However, countryspecific contexts must be considered in national policy discussions.

The neonatal period (birth to 28 completed days of life) is known as the most critical time for child survival ${ }^{1}$. During the Millennium Development Goal (MDG) era, decline in neonatal deaths was much slower than the decline in under-five mortality rate. Globally, the number of neonatal deaths has reduced by $39 \%$ from 4.3 to 2.8 million between 1990 and 2015; while the number of post-neonatal and childhood deaths (after 28 days to 5 years old) has declined by $61 \%$ from 7.7 to 3.3 million during this period ${ }^{2}$. The Sustainable Development Goal (SDG) aims to reduce neonatal mortality rate (NMR) to less than 12 per 1000 live births by $2030^{3}$. To reduce preventable neonatal deaths in the SDG era, it is critical to identify high-risk pregnancies. A rich volume of studies have identified various risk factors of neonatal mortality, including low socioeconomic status, insufficient antenatal care, and low birth weight ${ }^{4-6}$. Yet, only a few studies have assessed the potential importance of maternal history of poor pregnancy outcome. According to our review (up to the date of September 22nd, 2020), only eight studies, which were all at country- or sub country-levels, considered poor pregnancy outcomes as a risk factor of subsequent neonatal deaths; six of the eight studies were low- and middle-income countries, including India, Ethiopia, Bangladesh, and Ghana ${ }^{7-14}$. Previous studies have consistently found maternal history of neonatal deaths to be a significant factor associated with subsequent neonatal deaths $s^{9-14}$.

All the prior studies were conducted at national or sub-national levels with insufficient information to reveal whether this association is heterogeneous across countries. The identification of cross-country heterogeneity is essential for the design of global and national health agenda. If the association between history of neonatal deaths

\footnotetext{
${ }^{1}$ Vanke School of Public Health, Tsinghua University, Beijing, China. ${ }^{2}$ Economics and Planning Unit, Indian Statistical Institute (ISI), New Delhi, India. ${ }^{3}$ Division of Health Policy and Management, College of Health Sciences, Korea University, Seoul 02841, South Korea. ${ }^{4}$ Interdisciplinary Program in Precision Public Health, Department of Public Health Sciences, Graduate School of Korea University, Seoul, South Korea. ${ }^{5}$ Harvard Center for Population \& Development Studies, 9 Bow Street, Cambridge, MA, USA. ${ }^{6}$ Department of Social and Behavioral Sciences, Harvard T.H. Chan School of Public Health, Boston, MA, USA. ${ }^{\bowtie}$ email: rocklikim@korea.ac.kr; svsubram@ hsph.harvard.edu
} 
and subsequent neonatal mortality turns out to be consistent across countries, then history of neonatal death could be considered as one of the indicators to identify high risk pregnancies in LMICs ${ }^{10}$. On the other hand, if the association shows substantial heterogeneity across countries, then the implication would be to develop more localized guidelines to promote positive pregnancy.

In this paper, we pooled nationally representative data of Demographic and Health Surveys (DHS) from 56 countries to systematically assess the association between maternal history of neonatal death and risk of subsequent neonatal death. In addition to the pooled analysis, we present country-specific findings to inform the extent of heterogeneity across countries.

\section{Methods}

This project used publicly-accessible secondary data requested and downloaded from the DHS website (https:// dhsprogram.com/data/available-datasets.cfm). The DHS data are not collected specifically for this study and no one on the study team has access to identifiers linked to the data. These activities do not meet the regulatory definition of human subjects research. As such, IRB review is not required. The Harvard Longwood Campus IRB allows researchers to self-determine when their research does not meet the requirements for IRB oversight via guidance online regarding when an IRB application is required and the IRB Decision Tool.

Data source and study population. We pooled the most recent data for LMICs conducted between 2010 and 2018 from DHS, which collected detailed information on complete birth history, child information, and household characteristics from 15 to 49 years old women ${ }^{15}$. DHS used a multistage stratified sampling design, with the first stage generally involving choosing geographically-defined units such as villages for rural areas and census blocks for urban areas, and the second stage involving selecting the specific households or persons to be interviewed ${ }^{16}$. We excluded earlier survey rounds to avoid inconsistencies in the measurements, collection, and reporting of data required for this study.

There were 59 LMICs that had collected data on the history of neonatal deaths and subsequent neonatal mortality. We excluded Albania, Armenia, and Turkey from our analysis because the DHS data of these countries recorded less than 10 neonatal deaths within 5 years prior to the survey. The final sample included 56 LMICs. See Appendix Table 1 for a detailed list of countries and survey years included in the study.

There were a total of 602,587 live births recorded in the 56 LMICs. We excluded 156,978 live births from nulliparous women and 8560 nonsingleton live births. A total of 437,049 singleton live births from multiparous women aged 15 to 49 years were included in our analysis. Among all the live births included in the study, 6910 died during the neonatal period.

History of neonatal death. The primary predictor was maternal history of neonatal death. We identified these cases by examining the complete birth history of the mothers. A binary variable was constructed with the value of 1 representing a previous live birth that resulted in death during the first 28 completed days of life, and 0 otherwise.

Outcomes. Our main outcome was subsequent neonatal mortality. Following the World Health Organization (WHO) definition, neonatal death refers to the "deaths among live births during the first 28 completed days of life"17. For additional analyses, we define early neonatal death as "death among live births between 0 and 7 completed days of birth" and late neonatal death as "death among live births after 7 days to 28 completed days of birth" as our secondary outcomes ${ }^{18}$.

Covariates. In the adjusted analysis, we followed previous studies ${ }^{7-14}$ and controlled for a comprehensive set of covariates that are known to be associated with neonatal mortality, including socioeconomic and household factors, child factors, and pregnancy-related characteristics. The socioeconomic and household factors included household wealth quintiles, place of residence, maternal education, improved water, improved sanitation, and number of household members. Household wealth quintile was constructed by the DHS based on a selected set of household assets ${ }^{19}$. Place of residence was a dichotomous variable, which categorized the population into urban and rural. We classified maternal education to six categories (no schooling, $<5$ years, 5-7 years, 8-9 years, 10-11 years, and 12 years or more $)^{10}$. Water source was considered to be improved if the household had access to water piped into dwelling or yard/plot, public tap/standpipe, tube well or borehole, protected well or spring, rain water, and bottled water ${ }^{20}$. We considered sanitation facility to be improved if the household had access to flush to piped sewer system, septic tank, or pit latrine, ventilated improved pit latrine, pit latrine with slab, and composting toilet ${ }^{20}$. We divided number of household members to three categories $(<6,6-10,11$ and more). The child factors included sex of the child, birth weight of the child by type of report, size at birth, and breastfeeding initiation. We generated five categories for child birth weight by type of report, which were (1) not weighted, $(2)<2500 \mathrm{~g}$ based on mother's recall, (3) $<2500 \mathrm{~g}$ based on written card, (4) $\geq 2500 \mathrm{~g}$ based on mother's recall, and $(5) \geq 2500 \mathrm{~g}$ based on written card ${ }^{10}$. DHS classified child size at birth into three categories: (1) within reference range or higher, (2) small, (3) very small ${ }^{21}$. We generated a dichotomous variable to see whether breastfeeding was initiated $<1 \mathrm{~h}$ of birth ${ }^{22}$. The pregnancy-related covariates included number of antenatal cares, full tetanus protection, whether delivered with a skilled birth attendant (SBA), whether delivered in a health facility, maternal age at birth, and birth interval ${ }^{10}$. Global guidelines recommend frequent medical visits during the antenatal period to decrease the risk of birth complications, with recommendation ranging from at least four to optimally eight visits during the pregnancy ${ }^{23}$. We classified the number of antenatal cares into four categories (i.e. 0 times, 1-4 times, 5-7 times, 8 times or more). Maternal age at birth was classified in three categories $(<18,18-34,35$ years or older $)$ because both young and old maternal age are associated with higher risk of neo- 
natal mortality ${ }^{24}$. Similarly, birth interval was classified in three categories $(<18,18-59,60$ months and more) because long inter-pregnancy intervals (possibly longer than 5 years) and short intervals are independently associated with adverse pregnancy outcomes ${ }^{25}$. All other pregnancy-related covariates were dichotomized. For observations with missing data on one or more covariates we adopted the MI commands in STATA for multiple imputations ${ }^{26-28}$.

Statistical analysis. First, we examined the characteristics of the sample by their history of neonatal death. Second, we assessed the proportions of mothers with history of neonatal deaths among all multiparous women with previous live births in each of the 56 LMICs. To calculate the proportion, we performed crude logistic regression without any covariates. Third, we quantified the proportion of all neonatal deaths that have occurred to mothers with history of neonatal deaths using unadjusted logistic regression model.

To assess the association between mothers with history of neonatal death and subsequent neonatal mortality, we performed both pooled analysis and separate analysis for each country. We included sampling weight, clustering, and stratification variables provided by DHS to ensure that the estimates were representative at national level and in pooled analyses ${ }^{29}$. We clustered the sample at PSU level, which allows for interdependence of error terms within clusters and households ${ }^{29}$. In pooled analyses, we reweighted observations by country's population size, and included country-fixed effects to account for the unobservable country-level factors.

For both pooled and country-specific analyses, we developed three sets of logistic models to assess the association between maternal history of neonatal death and subsequent neonatal mortality. First, we ran a crude logistic model without controlling for any covariate to obtain unadjusted odds ratio (UOR) for subsequent neonatal mortality. Second, we ran a set of logistic models controlling each covariate separately to obtain partially adjusted odds ratio (PAOR). Third, we controlled for all the covariates introduced above to obtain fully adjusted odds ratio (FAOR). We calculated the degree to which OR attenuated after covariate adjustment using the formula $\frac{U O R-P A O R}{U O R-1} * 100$ or $\frac{U O R-F A O R}{U O R-1} * 100^{30}$.

We tested for consistency in the association by performing a series of stratified analyses defined by household wealth, place of residence, maternal education, sex of child, birth weight by report type, birth size, numbers of antenatal care visits, delivery with SBA, institutional delivery, full tetanus protection, and maternal age at birth. We also conducted two sets of supplementary analysis. First, we examined the association between maternal history of neonatal death with subsequent early neonatal mortality and late neonatal mortality, respectively. Second, we divided the history of neonatal death further into history of multiple neonatal deaths (more than one) and history of one neonatal death, and compared the effects between these two groups. We ran both unadjusted and adjusted logistic regressions for the supplementary analyses.

We used Stata (version 14.2) for all analyses procedures. All statistical tests were two-sided and $\mathrm{p}<0.05$ was considered to determine statistical significance.

\section{Results}

Descriptive summary. Of the 437,049 singleton live births included in the analysis, 37,290 (8.5\%) live births were by mothers with history of neonatal deaths. The NMR among mothers with history of neonatal death was 42.1 (95\% confidence interval [CI]: 39.6, 44.6) per 1000 live births, which was higher than those without history of neonatal deaths (13.2 per 1000 live births, 95\% CI 12.8, 13.7). Compared to mothers without history of neonatal deaths, mothers with such history were more likely to live in poorer households, in rural areas, with lower education, with babies unweighted or weighted low at birth, have small babies, have less numbers of antenatal care visits, deliver babies without a SBA, deliver babies at home, give births at older ages, have shorter birth intervals, without improved sanitation facility, without improved water access, initiate breastfeeding after $1 \mathrm{~h}$ of birth, and have more household members (Table 1).

The proportion of multiparous women with history of neonatal death varied across countries: only $2.4 \%$ $(95 \%$ CI 1.4 3.4) of the multiparous women had a previous history of neonatal death in Maldives, followed by Jordan $(2.6 \%, 95 \%$ CI 2.0, 3.2), and Colombia (3.0\%, 95\% CI 2.3, 3.6). Countries with the highest proportions were Ethiopia $(14.5 \%, 95 \%$ CI 12.9, 16.0), Niger $(13.2 \%, 95 \%$ CI 12.1, 14.4), and Sierra Leone $(12.7 \%$, $95 \%$ CI 11.6, 13.9) (Fig. 1).

For all 6910 neonatal deaths recorded among the most recent live births, 1549 (22.4\%) occurred to mothers with previous history of neonatal deaths. Countries with the lowest proportions were Dominican Republic (1.2\%, 95\% CI 0.0, 2.6), Congo, Rep. (7.1\%, 95\% CI 0.0, 16.4), and Kyrgyz Republic (7.7\%, 95\% CI 0.0, 16.0); while countries with the highest proportions were Niger (38.1\%, 95\% CI 26.0, 50.1), Ghana (33.4\%, 95\% CI 15.0, 51.9), and Angola (32.0\%, 95\% CI 20.3, 43.7) (Fig. 2).

Pooled analyses. In our pooled analysis, we found a strong association between maternal history of neonatal death and subsequent neonatal mortality, albeit FAOR attenuated by $50.7 \%$ compared to UOR. In the fully adjusted logistic regression models, history of neonatal death was associated with 2.1 (95\% CI 1.9, 2.4) higher odds of subsequent neonatal mortality, and this remained consistent across different subgroups with only one exception - for the subgroup of very small babies at birth, although history of neonatal death was significantly associated with higher odds of subsequent neonatal mortality in the unadjusted model $(\mathrm{UOR}=2.25,95 \% \mathrm{CI}$ $1.75,2.88)$; after covariate adjustment, FAOR was estimated to be $1.00(95 \%: 0.64,1.57)$, which was not statistically significant (Table 2).

When compared to estimates from partially adjusted models, the timing of breastfeeding initiation had the largest attenuation effect, causing the coefficient of history of neonatal death to reduce by $20.9 \%$ from an UOR: 3.25 (95\% CI 3.03, 3.50) to a PAOR: 2.78 (95\% CI 2.47, 3.13) (Appendix Table 2). This was followed by covariates on birth interval, birth weight and maternal age at birth, which attenuated the UOR by $6-10 \%$ each. 


\begin{tabular}{|c|c|c|c|}
\hline \multirow[b]{2}{*}{ Characteristics } & \multicolumn{3}{|c|}{$\begin{array}{l}\text { History of neonatal death (percentage or 95\% } \\
\text { CI) }\end{array}$} \\
\hline & Yes $(n=37,290)$ & No $(n=399,759)$ & P value \\
\hline Neonatal mortality per 1000 live births & $42.1(39.6,44.6)$ & $13.2(12.8,13.7)$ & $<0.001$ \\
\hline \multicolumn{4}{|l|}{ Wealth, quintile } \\
\hline Poorest & $12,239(32.8 \%)$ & $104,507(25.9 \%)$ & \multirow{5}{*}{$<0.001$} \\
\hline Poorer & $9009(24.2 \%)$ & $91,198(22.6 \%)$ & \\
\hline Middle & $7058(18.9 \%)$ & $79,983(19.8 \%)$ & \\
\hline Richer & $5546(14.9 \%)$ & $70,300(17.4 \%)$ & \\
\hline Richest & $3438(9.2 \%)$ & $58,010(14.4 \%)$ & \\
\hline \multicolumn{4}{|l|}{ Place of residence } \\
\hline Urban & $8546(22.9 \%)$ & $122,672(30.7 \%)$ & \multirow{2}{*}{$<0.001$} \\
\hline Rural & $28,744(77.1 \%)$ & $277,087(69.3 \%)$ & \\
\hline \multicolumn{4}{|l|}{ Maternal education, $y$} \\
\hline 0 & $18,339(49.2 \%)$ & $139,550(34.9 \%)$ & \multirow{7}{*}{$<0.00$} \\
\hline$<5$ & $4372(11.7 \%)$ & $42,876(10.7 \%)$ & \\
\hline $5-7$ & $6229(16.7 \%)$ & $74,132(18.5 \%)$ & \\
\hline $8-9$ & $3326(8.9 \%)$ & $48,505(12.1 \%)$ & \\
\hline $10-11$ & $1839(4.9 \%)$ & $37,466(9.4 \%)$ & \\
\hline$\geq 12$ & $2260(6.1 \%)$ & $49,368(12.4 \%)$ & \\
\hline Missing & $925(2.5 \%)$ & $7862(2.0 \%)$ & \\
\hline \multicolumn{4}{|l|}{ Sex of child } \\
\hline Male & $19,096(51.2 \%)$ & $208,651(52.2 \%)$ & \multirow{2}{*}{0.02} \\
\hline Female & $18,194(48.8 \%)$ & $191,108(47.8 \%)$ & \\
\hline \multicolumn{4}{|l|}{ Birth weight } \\
\hline Not weighted & $14,875(39.9 \%)$ & $122,203(30.6 \%)$ & \multirow{8}{*}{$<0.001$} \\
\hline Low & & & \\
\hline Mother's recall & $1595(4.3 \%)$ & $14,988(3.8 \%)$ & \\
\hline Written card & $1090(2.9 \%)$ & $11,202(2.8 \%)$ & \\
\hline Within reference range or higher & & & \\
\hline Mother's recall & $8518(22.8 \%)$ & $110,875(27.7 \%)$ & \\
\hline Written card & $7829(21.0 \%)$ & $99,798(25.0 \%)$ & \\
\hline Missing & $3383(9.1 \%)$ & $40,693(10.2 \%)$ & \\
\hline \multicolumn{4}{|l|}{ Birth size } \\
\hline Within reference range or higher & $29,172(78.2 \%)$ & $318,505(79.7 \%)$ & \multirow{4}{*}{$<0.001$} \\
\hline Small & $4527(12.1 \%)$ & $40,049(10.0 \%)$ & \\
\hline Very small & $1997(5.4 \%)$ & $14,988(3.8 \%)$ & \\
\hline Missing & $1594(4.3 \%)$ & $26,217(6.6 \%)$ & \\
\hline \multicolumn{4}{|l|}{ Antenatal care visits, no } \\
\hline 0 & $6931(18.6 \%)$ & $61,171(15.3 \%)$ & \multirow{5}{*}{$<0.001$} \\
\hline $1-4$ & $18,525(49.7 \%)$ & $180,712(45.2 \%)$ & \\
\hline $5-7$ & $7643(20.5 \%)$ & $93,458(23.4 \%)$ & \\
\hline$\geq 8$ & $3707(9.9 \%)$ & $58,795(14.7 \%)$ & \\
\hline Missing & $484(1.3 \%)$ & $5623(1.4 \%)$ & \\
\hline \multicolumn{4}{|l|}{ Delivered with skilled birth attendant } \\
\hline Yes & $18,743(50.3 \%)$ & $230,425(57.6 \%)$ & \multirow{3}{*}{$<0.001$} \\
\hline No & $18,494(49.6 \%)$ & $169,021(42.3 \%)$ & \\
\hline Missing & $53(0.1 \%)$ & $313(0.1 \%)$ & \\
\hline Institutional delivery & & & \\
\hline Yes & $21,953(58.9 \%)$ & $266,231(66.6 \%)$ & $<0001$ \\
\hline No & $15,337(41.1 \%)$ & $133,528(33.4 \%)$ & $<0.001$ \\
\hline Full tetanus protection & & & \\
\hline Yes & $27,082(72.6 \%)$ & $290,386(72.6 \%)$ & 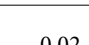 \\
\hline No & $10,208(27.4 \%)$ & $109,373(27.4 \%)$ & 0.02 \\
\hline Maternal age at birth, $y$ & & & \\
\hline$<18$ & $310(0.8 \%)$ & $3447(0.9 \%)$ & \\
\hline $18-34$ & $27,486(73.7 \%)$ & $332,008(83.1 \%)$ & $<0.001$ \\
\hline$\geq 35$ & $9494(25.5 \%)$ & $64,304(16.1 \%)$ & \\
\hline Continued & & & \\
\hline
\end{tabular}




\begin{tabular}{|c|c|c|c|}
\hline \multirow[b]{2}{*}{ Characteristics } & \multicolumn{3}{|c|}{$\begin{array}{l}\text { History of neonatal death (percentage or } 95 \% \\
\text { CI) }\end{array}$} \\
\hline & Yes $(n=37,290)$ & No $(n=399,759)$ & $P$ value \\
\hline \multicolumn{4}{|c|}{ Birth interval, mo } \\
\hline$<18$ & $5342(14.3 \%)$ & $25,893(6.5 \%)$ & \multirow{3}{*}{$<0.001$} \\
\hline $18-59$ & $27,116(72.7 \%)$ & $296,804(74.3 \%)$ & \\
\hline$\geq 60$ & $4832(13.0 \%)$ & $77,062(19.3 \%)$ & \\
\hline \multicolumn{4}{|c|}{ Improved sanitation } \\
\hline Yes & $14,200(38.1 \%)$ & $194,851(48.7 \%)$ & \multirow{3}{*}{$<0.001$} \\
\hline No & $21,103(56.6 \%)$ & $184,093(46.1 \%)$ & \\
\hline Missing & $1987(5.3 \%)$ & $20,815(5.2 \%)$ & \\
\hline \multicolumn{4}{|l|}{ Improved water } \\
\hline Yes & $23,733(63.6 \%)$ & $261,898(65.5 \%)$ & \multirow{3}{*}{$<0.001$} \\
\hline No & $23,733(29.7 \%)$ & $110,623(27.7 \%)$ & \\
\hline Missing & $2479(6.7 \%)$ & $27,238(6.8 \%)$ & \\
\hline \multicolumn{4}{|c|}{ Breastfeeding initiation $<1 \mathrm{~h}$ of birth } \\
\hline Yes & $16,043(43.0 \%)$ & $191,803(48.0 \%)$ & \multirow{3}{*}{$<0.001$} \\
\hline No & $18,577(49.8 \%)$ & $180,600(45.2 \%)$ & \\
\hline Missing & $2670(7.2 \%)$ & $27,356(6.8 \%)$ & \\
\hline \multicolumn{4}{|c|}{ Number of household members } \\
\hline$<6$ & $14,072(37.7 \%)$ & $164,684(41.2 \%)$ & \multirow{3}{*}{$<0.001$} \\
\hline $6-10$ & $18,420(49.4 \%)$ & $189,339(47.4 \%)$ & \\
\hline$\geq 11$ & $4798(12.9 \%)$ & $45,736(11.4 \%)$ & \\
\hline
\end{tabular}

Table 1. Characteristics of the multiparous women stratified by history of neonatal death.

Country-specific analyses. The unadjusted and fully adjusted associations between history of neonatal death and subsequent neonatal mortality for each country are presented in Fig. 3 and Appendix Table 3. The FAOR varied from 0.4 (95\% CI 0.0, 4.0) in Dominican Republic to 16.1 (95\% CI 3.6, 42.0) in South Africa. We found 40 of the 56 countries with statistically significant association between history of neonatal death and subsequent neonatal mortality. The top five countries with the largest FAOR were South Africa, Egypt (11.7, 95\% CI 4.5, 39.7), Jordan (10.7, 95\% CI 3.5, 32.9), Philippines (6.2, 95\% CI 2.6, 15.0), and Tajikistan (6.0, 95\% CI $2.1,16.8)$. There were 16 countries with statistically insignificant association between history of neonatal death and subsequent neonatal mortality using the adjusted model (e.g. Bangladesh, Burundi, Gabon). (1) We fully adjusted the models with factors related to socioeconomic environment, maternal anthropometry, and pregnancy care. (2) We excluded Albania and Armenia as they have less than 20 observations with recorded maternal history of neonatal death. (3) Bangladesh, Kyrgyz Republic, Tajikistan, and Turkey do not have valid data on full tetanus protection. Therefore, we excluded this covariate from the model. (4) Yemen does not have valid data on maternal education. Therefore, we excluded this covariate from the model.

The FAORs attenuated by more than $10 \%$ in 39 of the 56 countries compared to their respective UORs, with the largest attenuation occurring in Kyrgyz Republic (change: 128.1\%, UOR: 2.0, FAOR: 0.7), followed by Comoros (change: 80.0\%, UOR: 6.9, FAOR: 2.2) and Gabon (change: 64.7\%, UOR: 4.0; FAOR: 2.1) (Appendix Table 3).

Supplementary analysis. We performed two sets of supplementary analysis. First, we examined whether history of neonatal death had a stronger association with subsequent neonatal mortality in the earlier period (i.e. 0 and 7 completed days of birth) than in the later period (i.e. after 7 days to 28 days of birth). With the pooled sample, we found the history of neonatal death to be associated with 2.7 (95\% CI 2.5, 3.0) higher odds of subsequent early neonatal death using the fully adjusted model, which was larger than the association for subsequent late neonatal death (FAOR: 1.8, 95\% CI 1.5, 2.2) (Appendix Figure 1). Second, we investigated whether history of multiple neonatal death has a stronger association than history of single neonatal death. We found that the association was FAOR: 2.8 (95\% CI 2.3, 3.5) among those with multiple previous neonatal deaths, and FAOR: 1.9 (95\% CI 1.7, 2.3) for those with only one previous neonatal death (Appendix Figure 2).

In the country-level analysis, we found that among the 42 countries with sufficient observations (more than 20 deaths) for both early neonatal and late neonatal periods, there were 25 countries with the associations being stronger for subsequent early neonatal mortality than for subsequent late neonatal mortality. For example, in Gambia, the history of neonatal deaths was associated with 4.6 (95\% CI 2.1, 10.2) times higher odds of subsequent early neonatal death, which was greater than that for subsequent late neonatal death at 0.7 (95\% CI $0.1,4.0)$ (Appendix Table 4). Furthermore, 39 of the 50 countries with sufficient observations had stronger associations among mothers with multiple previous neonatal deaths than those with only one previous neonatal death. The difference between the two groups reached statistically significance $(\mathrm{p}<0.01)$ in 9 countries, including Bangladesh, Egypt, Haiti, India, Kenya, Mali, Nepal, South Africa, and Togo (Appendix Table 5). 


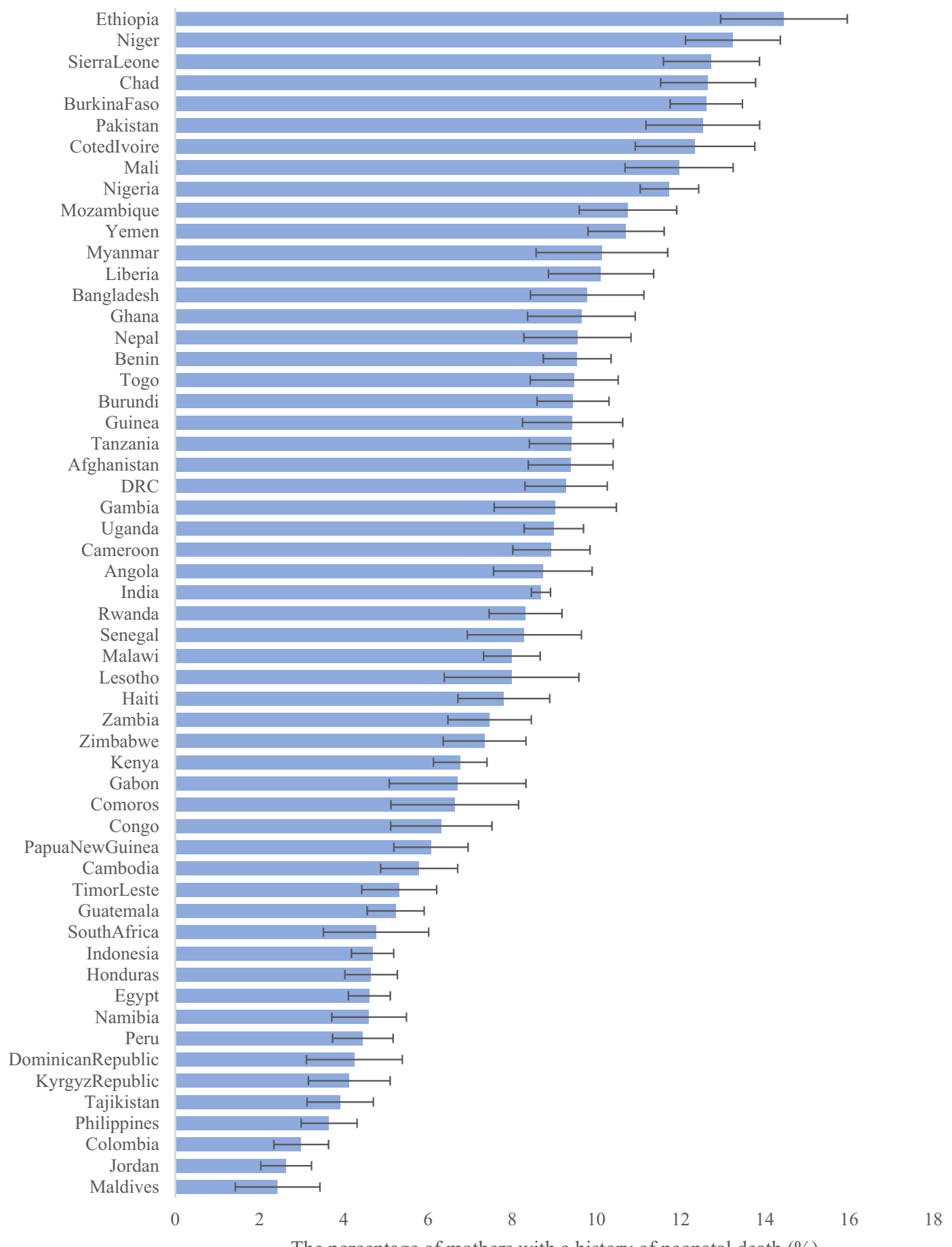

Figure 1. Between-country variations in percent of mothers with history of neonatal death.

\section{Discussion}

Four salient findings emerged from our analysis using the most recent data from 56 LMICs. First, the proportion of mothers with history of neonatal death varied widely across countries from 2 to $15 \%$. We also found substantial cross-country variation in the proportion of neonatal deaths that has occurred to mothers with history of neonatal death, ranging from 1 to $38 \%$. Second, we found maternal history of neonatal deaths to be strongly associated with subsequent neonatal deaths in both pooled and a series of subgroup analyses, even after adjusting for a comprehensive set of covariates. Third, at the country-level, we found the associations between maternal history of neonatal death and subsequent neonatal death to vary largely across countries. The FAOR ranged between 0.4 and 16.1 , yet the majority of countries had statistically significant association. The attenuation between UOR and FAOR was the largest in Kyrgyz Republic, Comoros, and Gabon. Lastly, the association 


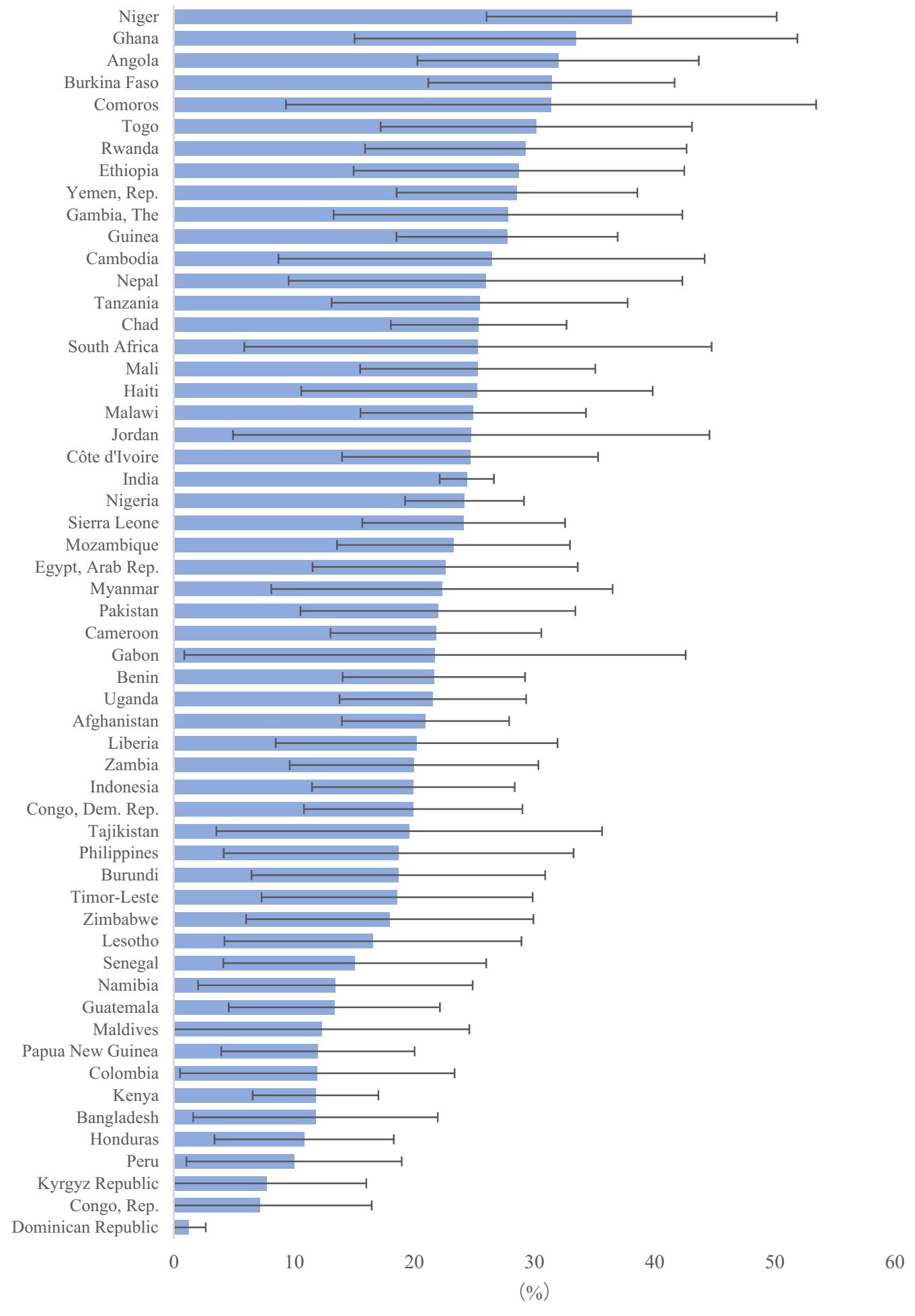

Figure 2. The percentage of neonatal deaths occurring to mothers with history of neonatal death.

between history of neonatal deaths and subsequent neonatal mortality was stronger in the earlier neonatal periods (i.e. 0-7 completed days) in both the pooled and most country-level analyses. Moreover, the association was stronger among mothers with multiple previous neonatal deaths than those with one previous neonatal death.

The large variation in the proportions of mothers with history of neonatal death suggests that with appropriate interventions, the prevalence of history of neonatal deaths in many countries can be potentially modified. For example, in Maldives, only 2\% of the women had one or more previous neonatal deaths, and this is closely related to the country's continuous efforts to tackle maternal and child health in the past two decades. Health 


\begin{tabular}{|c|c|c|c|}
\hline Variable & UOR (95\% CI) & FAOR $(95 \% \mathrm{CI})$ & Change (\%) \\
\hline Overall & $3.25(3.03,3.50)$ & $2.11(1.86,2.40)$ & 50.7 \\
\hline \multicolumn{4}{|l|}{ Wealth, quintile } \\
\hline Poorest & $3.08(2.71,3.49)$ & $2.36(1.90,2.93)$ & 34.6 \\
\hline Poorer & $3.16(2.74,3.63)$ & $1.89(1.48,2.41)$ & 58.8 \\
\hline Middle & $3.36(2.84,3.96)$ & $2.17(1.63,2.88)$ & 50.4 \\
\hline Richer & $3.11(2.58,3.75)$ & $1.66(1.18,2.33)$ & 68.7 \\
\hline Richest & $3.50(2.74,4.46)$ & $2.18(1.26,3.78)$ & 52.8 \\
\hline \multicolumn{4}{|l|}{ Place of residence } \\
\hline Urban & $3.44(2.95,4.01)$ & $1.85(1.31,2.62)$ & 65.2 \\
\hline Rural & $3.17(2.92,3.44)$ & $2.17(1.89,2.49)$ & 46.1 \\
\hline \multicolumn{4}{|l|}{ Maternal education, $y$} \\
\hline 0 & $2.86(2.58,3.17)$ & $2.24(1.90,2.65)$ & 33.3 \\
\hline$<5$ & $2.86(2.58,3.17)$ & $2.24(1.59,3.15)$ & 33.3 \\
\hline $5-7$ & $3.49(2.85,4.27)$ & $1.49(1.06,2.10)$ & 80.3 \\
\hline $8-9$ & $2.94(2.45,3.52)$ & $2.00(1.30,3.08)$ & 48.5 \\
\hline $10-11$ & $3.24(2.55,4.12)$ & $2.58(1.23,5.42)$ & 29.5 \\
\hline$\geq 12$ & $3.81(2.74,5.29)$ & $2.39(1.21,4.72)$ & 50.5 \\
\hline \multicolumn{4}{|l|}{ Sex of child } \\
\hline Male & $3.23(2.93,3.56)$ & $1.97(1.65,2.36)$ & 56.5 \\
\hline Female & $3.32(2.98,3.69)$ & $2.28(1.90,2.73)$ & 44.8 \\
\hline \multicolumn{4}{|l|}{ Birth weight } \\
\hline Not weighted & $2.84(2.55,3.15)$ & $2.21(1.83,2.65)$ & 34.2 \\
\hline \multicolumn{4}{|l|}{ Low } \\
\hline Mother's recall & $2.23(1.71,2.92)$ & $1.47(1.09,2.06)$ & 94.3 \\
\hline Written card & $2.08(1.20,3.61)$ & $1.63(1.01,2.67)$ & 85.2 \\
\hline \multicolumn{4}{|l|}{ Within reference range or higher } \\
\hline Mother's recall & $3.32(2.81,3.93)$ & $2.45(1.87,3.21)$ & 37.5 \\
\hline Written card & $3.47(2.65,4.56)$ & $2.16(1.44,3.26)$ & 53.0 \\
\hline \multicolumn{4}{|l|}{ Birth size } \\
\hline Within reference range or higher & $3.37(3.10,3.68)$ & $2.37(2.06,2.74)$ & 42.2 \\
\hline Small & $2.48(2.04,3.01)$ & $1.65(1.13,2.40)$ & 56.1 \\
\hline Very small & $2.25(1.75,2.88)$ & $1.00(0.64,1.57)$ & 100.0 \\
\hline \multicolumn{4}{|l|}{ Antenatal care visits, no } \\
\hline 0 & $4.14(3.08,5.55)$ & $2.01(1.58,2.56)$ & 67.8 \\
\hline $1-4$ & $2.96(2.54,3.46)$ & $2.17(1.81,2.61)$ & 40.3 \\
\hline $5-7$ & $3.01(2.71,3.34)$ & $1.85(1.36,2.53)$ & 57.7 \\
\hline$\geq 8$ & $3.38(2.88,3.98)$ & $2.80(1.73,4.55)$ & 24.4 \\
\hline \multicolumn{4}{|c|}{ Delivered with skilled birth attendant } \\
\hline Yes & $3.82(2.98,4.90)$ & $2.03(1.70,2.43)$ & 63.5 \\
\hline No & $3.44(3.12,3.80)$ & $2.19(1.82,2.62)$ & 51.2 \\
\hline \multicolumn{4}{|l|}{ Institutional delivery } \\
\hline Yes & $3.01(2.70,3.36)$ & $2.19(1.85,2.60)$ & 40.8 \\
\hline No & $3.44(3.13,3.77)$ & $2.01(1.66,2.44)$ & 58.6 \\
\hline \multicolumn{4}{|l|}{ Full tetanus protection } \\
\hline Yes & $2.89(2.57,3.24)$ & $2.16(1.85,2.51)$ & 38.6 \\
\hline No & $3.24(2.97,3.53)$ & $2.02(1.60,2.56)$ & 54.5 \\
\hline \multicolumn{4}{|l|}{ Maternal age at birth, $y$} \\
\hline$<18$ & $3.27(2.87,3.73)$ & $2.02(2.02,2.02)$ & 55.1 \\
\hline $18-34$ & $3.26(2.99,3.55)$ & $2.05(1.76,2.40)$ & 53.5 \\
\hline$\geq 35$ & $2.71(2.38,3.10)$ & $2.29(1.82,2.88)$ & 24.6 \\
\hline \multicolumn{4}{|l|}{ Birth interval, mo } \\
\hline$<18$ & $3.15(2.66,3.73)$ & $1.92(1.45,2.54)$ & 57.2 \\
\hline $18-59$ & $3.11(2.85,3.39)$ & $2.25(1.93,2.63)$ & 40.8 \\
\hline$\geq 60$ & $2.60(2.13,3.19)$ & $1.60(1.06,2.42)$ & 62.5 \\
\hline \multicolumn{4}{|l|}{ Improved sanitation } \\
\hline Yes & $2.84(2.58,3.12)$ & $2.04(1.75,2.39)$ & 43.5 \\
\hline \multicolumn{4}{|l|}{ Continued } \\
\hline
\end{tabular}




\begin{tabular}{|l|l|l|l|}
\hline Variable & UOR $(\mathbf{9 5} \% \mathbf{C I})$ & FAOR $(95 \%$ CI $)$ & Change (\%) \\
\hline No & $3.63(3.22,4.08)$ & $2.23(1.78,2.80)$ & 53.2 \\
\hline Improved water & $2.90(2.53,3.32)$ & $1.93(1.54,2.43)$ & 51.1 \\
\hline Yes & $3.44(3.15,3.76)$ & $2.20(1.89,2.56)$ & 50.8 \\
\hline No & $2.57(2.18,3.01)$ & $1.96(1.66,2.33)$ & 38.9 \\
\hline Breastfeeding initiation < 1 h of birth \\
\hline Yes & $3.05(2.58,3.62)$ & $2.28(1.88,2.76)$ & 37.6 \\
\hline No & $3.43(3.10,3.79)$ & $1.87(1.55,2.25)$ & 64.2 \\
\hline Number of household members & $3.06(2.73,3.44)$ & $2.16(1.76,2.65)$ & 43.7 \\
\hline$<6$ & $3.84(3.07,4.79)$ & $3.13(2.20,4.44)$ & 25.0 \\
\hline $6-10$ & &
\end{tabular}

Table 2. Unadjusted odds ratio (UOR) and fully adjusted odds ratios (FAOR) for neonatal mortality by maternal history of neonatal death, pooled sample and subgroup samples. (1) "Change" is the degree to which the odds ratios were attenuated after adjustment that was calculated according to the formula $\frac{U O R-F A O R}{U O R-1} * 100$.

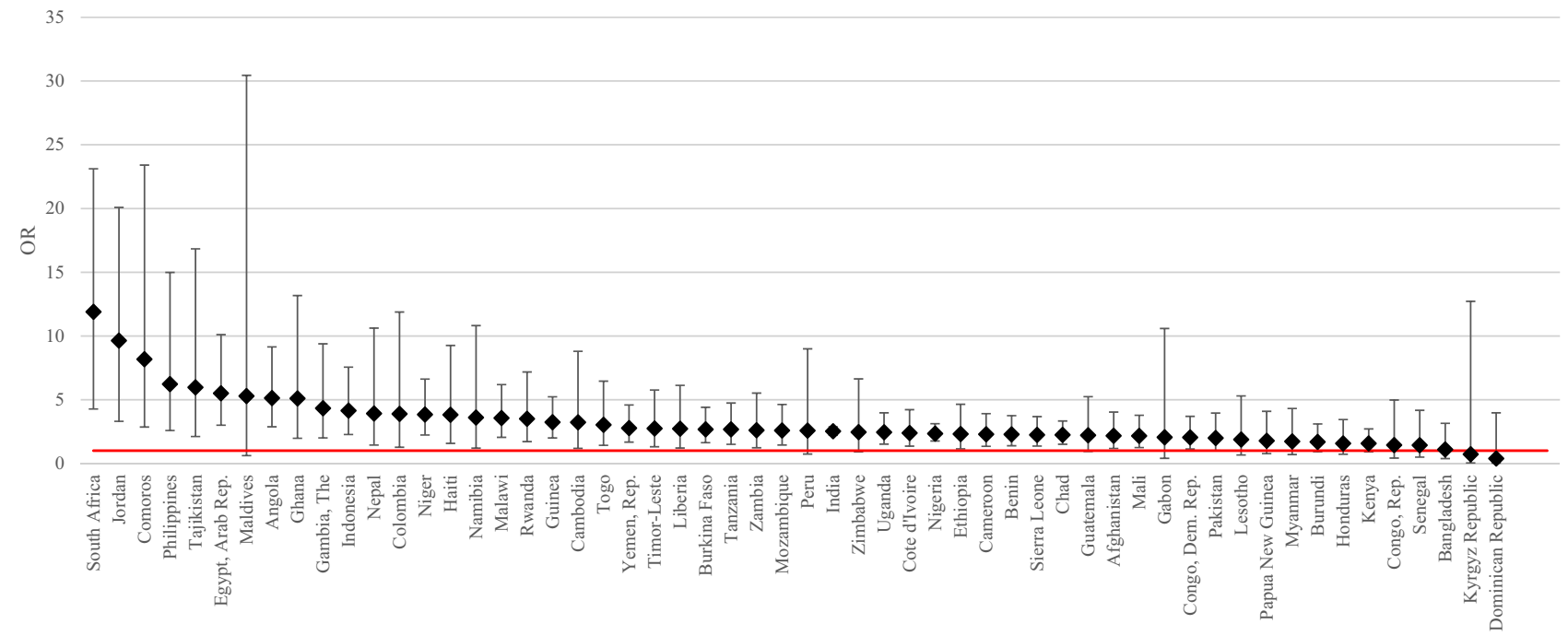

Figure 3. Fully adjusted odds ratios (FAOR) for neonatal mortality by maternal history of neonatal death, by country.

workers in Maldives received additional training and were deployed to provide home visits and close monitoring, particularly for women with high-risk pregnancies. Health officials, along with international organizations (e.g. World Health Organization, United Nations Population Fund, etc.), expanded emergency obstetric care availability and family planning access to ensure all pregnancies were wanted and were taken proper care of ${ }^{31,32}$. The Health Master Plan for 2006-2015 put a strong focus on the newborn health in Maldives, which was further strengthened by the Maldives Every Newborn Action Plan (2014) with a commitment to end all preventable newborn deaths ${ }^{32}$. As a result, NMR in Maldives reduced by $89 \%$ from 42 per 1000 live births in 1990 to 5 in $2016^{33}$.

Our study also found a wide variation in the proportions of neonatal deaths that have occurred to mothers with one or more previous neonatal deaths. In Niger, among all newborns who died within 28 completed days of birth, almost two fifths of them happened to mothers with history of neonatal death. Two reasons could partially explain this striking variation. First, since a larger proportion of the Nigerien mothers (13\%) experienced death of newborns before, it was more likely for a child to be born to these mothers. Second, other risk factors, such as poor socioeconomic status, obstetric and pediatric resources available to the households, and the related health behavior during antenatal and intrapartum periods, are likely to persist and constantly affect pregnancies at different times ${ }^{9-14}$. However, it is notable that even after we adjusted for a rich set of covariates, the association remained statistically significant. Similarly, we found that despite large attenuation, the association between maternal history of neonatal deaths and subsequent neonatal mortality remained strong in many countries, including Burkina Faso, Jordan, and Timor-Leste. This suggests that there might be either a direct causal link between the history of neonatal death and subsequent neonatal deaths or an indirect link via other unobservable residual confounding related to environmental factors, function of health care system, quality of care, and genetic, immunological, or etiological disorders ${ }^{10,34,35}$. 
Dominican Republic, Congo, Rep., and Kyrgyz Republic had less than $10 \%$ of the newborn deaths occurred to mothers with previous neonatal deaths, and the associations between previous neonatal deaths and subsequent newborn mortality were also weak. This may be a result of the countries' existing efforts to identify high-risk pregnancies. For example, in Kyrgyz Republic, attempts were made to ensure women's uptake of timely obstetric care from specialists, including the Promotion of Perinatal Health project that specially aimed to strengthen maternal and newborn referral system ${ }^{36}$. In Dominican Republic, community health workers have been trained to perform essential roles in identifying pregnancy complications and being responsible to report high risk patients to the doctors ${ }^{37,38}$.

Despite such scattered efforts in several countries, in general, most LMICs still lack specific programs or interventions to identify mothers with high-risk pregnancies who would benefit the most from intensive obstetric and pediatric surveillance and care during antenatal, intrapartum, and postpartum periods ${ }^{35,39-41}$. Maternal history of neonatal death, as a simple identification tool for high-risk pregnancy, can enhance timely referral, effective maternal counselling, early diagnosis and treatment of specific disorders, and access to higher-level health facilities for obstetric and pediatrician care ${ }^{35}$.

There are several limitations to this study. First, we were only able to include 56 LMICs with available data. Therefore, the estimates from our pooled sample were not representative at either global-level or by income groups. Second, we were unable to include previous stillbirth as part of the covariates because this indicator was not collected or specified as a separate category in majority of the countries. Third, the usage of observational data and cross-sectional analysis limit our capacity to make any causal inference. Although we have adjusted for numerous socioeconomic, child, and pregnancy-related factors, our analyses are still subject to confounders that are unobserved or without available data. There were some potential confounders that were not collected in DHS or with substantial amount of missing values, such as the quality of delivery care, the usage of insecticidetreated bed nets, and pregnancy complications. Fourth, despite the fact that we limited our data to the most recent surveys conducted in a relatively small range of time (between 2010 and 2018), the survey year of each country varied from each other which might be a challenge for a cross-sectional study. Last, although DHS has been widely adopted as the most reliable source on child mortality ${ }^{42-45}$, we recognize the potential data collection problems, including misreporting dates to birth or age at death ${ }^{46}$.

Using the most comprehensive and up-to-date data, our investigation indicates that adverse outcomes of previous pregnancies is a strong risk factor of future neonatal deaths in a majority of the studied countries. This finding suggests that maternal history of neonatal deaths could be a powerful and effective indicator to identify women with high-risk pregnancies, especially in LMICs. At the same time, the substantial heterogeneity across countries indicates the need for context-specific understanding to inform national policies and programs. Therefore, we call for more national and regional level studies for the design of localized interventions to identify high-risk pregnancies and reduce neonatal mortality.

Received: 15 April 2021; Accepted: 24 August 2021

Published online: 07 October 2021

\section{References}

1. World Health Organization. Neonatal mortality (WHO, 2017) https://www.who.int/gho/child_health/mortality/neonatal_text/ en/ (Accessed 8 June 2020).

2. Global Burden of Disease Collaborative Network. Global Burden of Disease Study 2017 (GBD 2017) Results (Institute for Health Metrics and Evaluation (IHME), 2018) http://ghdx.healthdata.org/gbd-results-tool (Accessed 19 Dec 2018).

3. United Nations. Sustainable Development Goals. https://www.un.org/sustainabledevelopment/health/ (Accessed 14 July 2018).

4. Black, R. E. et al. Reproductive, maternal, newborn, and child health: Key messages from Disease Control Priorities 3rd Edition. Lancet 388, 2811-2824 (2016).

5. Andegiorgish, A. K. et al. Neonatal mortality and associated factors in the specialized neonatal care unit Asmara, Eritrea. BMC Public Health 20, 10 (2020).

6. Lawn, J. E., Cousens, S. \& Zupan, J. 4 million neonatal deaths: When? where? why?. Lancet 365, 891-900 (2005).

7. Clegg, E. J. \& Cross, J. F. Aspects of neonatal death in St Kilda, 1830-1930. J. Biosoc. Sci. 26, 97-106 (1994).

8. Murdock, A. I. Factors associated with high-risk pregnancies in Canadian Inuit. Can. Med. Assoc. J. 120, 291-294 (1979).

9. Williams, E. K. et al. Birth interval and risk of stillbirth or neonatal death: Findings from rural north India. J. Trop. Pediatr. 54, 321-327 (2008).

10. Kapoor, M. et al. Association of maternal history of neonatal death with subsequent neonatal death in India. JAMA Netw. Open. 3, e202887 (2020).

11. Alam, N. \& David, P. H. Infant and child mortality in Bangladesh: Age-specific effects of previous child's death. J. Biosoc. Sci. 30, 333-348 (1998).

12. Yirgu, R., Molla, M. \& Sibley, L. Determinants of neonatal mortality in rural Northern Ethiopia: A population based nested case control study. PLoS ONE 12, e0172875 (2017).

13. Avoka, J. A., Adanu, R. M., Wombeogo, M., Seidu, I. \& Dun-Dery, E. J. Maternal and neonatal characteristics that influence very early neonatal mortality in the Eastern Regional Hospital of Ghana, Koforidua: A retrospective review. BMC. Res. Notes 11, 91 (2018).

14. Greenwood, R., Golding, J., McCaw-Binns, A., Keeling, J. \& Ashley, D. The epidemiology of perinatal death in Jamaica. Paediatr. Perinat. Epidemiol. 8(Suppl 1), 143-157 (1994).

15. USAID. The DHS Program-Available Datasets. https://www.dhsprogram.com/data/available-datasets.cfm (Accessed 25 July 2019).

16. Turner AG. Sampling strategies (2003) http://mdgs.un.org/unsd/demographic/meetings/egm/Sampling_1203/docs/no_2.pdf (Accessed 7 Nov 2019).

17. World health Organisation. Neonatal mortality rate (per 1000 live births). http://www.who.int/healthinfo/morttables (Accessed 7 Aug 2020).

18. Pathirana, J. et al. Neonatal death: Case definition \& guidelines for data collection, analysis, and presentation of immunization safety data. Vaccine. 34, 6027-6037 (2016). 
19. Demographic and Health Surveys. The DHS Program -Wealth Index Construction. http://www.dhsprogram.com/topics/wealthindex/Wealth-Index-Construction.cfm (Accessed $23 \mathrm{Feb} 2017$ ).

20. World Health Organization. Water sanitation hygiene. (WHO, 2012) https://www.who.int/water_sanitation_health/monitoring/ jmp2012/key_terms/en/ (Accessed 12 Sept 2019).

21. UNICEF. The DHS Program-DHS Survey Indicators-Characteristics of Households (2019) https://dhsprogram.com/data/DHSSurvey-Indicators-Characteristics-of-Households.cfm (Accessed 12 Sept 2019).

22. Kim, R. et al. Assessing associational strength of 23 correlates of child anthropometric failure: An econometric analysis of the 2015-2016 National Family Health Survey, India. Soc. Sci. Med. 238, 112374 (2019).

23. World Health Organisation. WHO recommendations on antenatal care for a positive pregnancy experience (2017) https://www. who.int/publications/i/item/9789241549912 (Accessed 18 Aug 2021).

24. Kim, Y. N., Choi, D. W., Kim, D. S., Park, E. C. \& Kwon, J. Y. Maternal age and risk of early neonatal mortality: A national cohort study. Sci. Rep. 11, 814 (2021).

25. Kangatharan, C., Labram, S. \& Bhattacharya, S. Interpregnancy interval following miscarriage and adverse pregnancy outcomes: Systematic review and meta-analysis. Hum. Reprod. Update 23, 221-231 (2017).

26. Kruk, M. E. et al. Mortality due to low-quality health systems in the universal health coverage era: A systematic analysis of amenable deaths in 137 countries. Lancet 392, 2203-2212 (2018).

27. Monden, C. W. S. \& Smits, J. Mortality among twins and singletons in sub-Saharan Africa between 1995 and 2014: A pooled analysis of data from 90 Demographic and Health Surveys in 30 countries. Lancet Glob. Health 5, e673-e679 (2017).

28. Li, Z., Kim, R., Vollmer, S. \& Subramanian, S. V. Factors associated with child stunting, wasting, and underweight in 35 low- and middle-income countries. JAMA Netw. Open. 3, e203386 (2020).

29. Kim, R., Mejía-Guevara, I., Corsi, D. J., Aguayo, V. M. \& Subramanian, S. V. Relative importance of 13 correlates of child stunting in South Asia: Insights from nationally representative data from Afghanistan, Bangladesh, India, Nepal, and Pakistan. Soc. Sci. Med. 2017(187), 144-154 (1982).

30. Subramanian, S. V. et al. The mortality divide in India: The differential contributions of gender, caste, and standard of living across the life course. Am. J. Public Health 96, 818-825 (2006).

31. United Nations Population Fund. Stunning plunge in maternal deaths recorded in Maldives (2016) https://www.unfpa.org/news/ stunning-plunge-maternal-deaths-recorded-maldives (Accessed 22 Sept 2020).

32. UNICEF. National Child Health Strategy - Every Newborn Action Plan (ENAP) Maldives (2016).

33. Organization WH. Maldives RMNCAH Factsheet (2018) https://www.who.int/docs/default-source/maternal-health/rmncah-fsmav.pdf?sfvrsn=70714afc_2.

34. Abdellatif, M. et al. The patterns and causes of neonatal mortality at a tertiary hospital in oman. Oman Med. J. 28, 422-426 (2013).

35. Khan, J. Y. \& Dookeran, K. A. Maternal history of neonatal death as an emerging risk factor of subsequent neonatal mortality in low- and middle-income countries. JAMA Netw. Open. 3, e202972 (2020).

36. GIZ. On the right track: Promotion of Perinatal Health in Kyrgyzstan. https://giz-health.kg/en/news/25-On-the-right-track.html (Accessed 22 Sept 2020).

37. CHWCentral. Community health workers and use of mHealth: improving identification of pregnancy complications and access to care in the Dominican Republic. https://chwcentral.org/resources/community-health-workers-and-use-of-mhealth-improvingidentification-of-pregnancy-complications-and-access-to-care-in-the-dominican-republic (Accessed 22 Sept 2020).

38. Project Hope Republica Dominicana. Providing Quality Health Care to Women and Children in the Dominican Republic. https:// www.projecthope.org/wp-content/uploads/2018/08/HOPE-DR-Clinics-2012.pdf.

39. Sun, L. et al. Estimation of high risk pregnancy contributing to perinatal morbidity and mortality from a birth population-based regional survey in 2010 in China. BMC Pregnancy Childbirth 14, 338 (2014).

40. Demitto, M. O., Gravena, A. A., Dell'Agnolo, C. M., Antunes, M. B. \& Pelloso, S. M. High risk pregnancies and factors associated with neonatal death. Revista da Escola de Enfermagem da U S P. 51, e03208 (2017).

41. Unterscheider, J. et al. Fetal growth restriction and the risk of perinatal mortality-case studies from the multicentre PORTO study. BMC Pregnancy Childbirth 14, 63 (2014).

42. Wang, H. et al. Global, regional, and national levels of neonatal, infant, and under-5 mortality during 1990-2013: A systematic analysis for the Global Burden of Disease Study 2013. Lancet 384, 957-979 (2014).

43. Rajaratnam, J. K. et al. Neonatal, postneonatal, childhood, and under-5 mortality for 187 countries, 1970-2010: A systematic analysis of progress towards Millennium Development Goal 4. Lancet 375, 1988-2008 (2010).

44. Wang, H. B. Z. et al. Global, regional, national, and selected subnational levels of stillbirths, neonatal, infant, and under-5 mortality, 1980-2015: A systematic analysis for the Global Burden of Disease Study 2015. Lancet 388, 1725-1774 (2016).

45. Li, Z., Li, M., Subramanian, S. V. \& Lu, C. Assessing levels and trends of child health inequality in 88 developing countries: From 2000 to 2014. Glob. Health Action 10, 1408385 (2017)

46. Mwale MW. Infant and child mortality. https://dhsprogram.com/pubs/pdf/fr175/08chapter08.pdf (Accessed 6 Oct 2018).

\section{Acknowledgements}

ZL had full access to all the data in the study and takes responsibility for the integrity of the data and the accuracy of the data analysis.

\section{Author contributions}

Conceptualization and design: S.V.S., R.K.; Data acquisition and analysis: Z.L.; Data interpretation: Z.L., M.K., R.K., S.V.S.; Drafting of the manuscript: Z.L.; Critical revisions to manuscript: Z.L., M.K., R.K., S.V.S.; Overall supervision: S.V.S., R.K.

\section{Competing interests}

The authors declare no competing interests.

\section{Additional information}

Supplementary Information The online version contains supplementary material available at https://doi.org/ 10.1038/s41598-021-97481-3.

Correspondence and requests for materials should be addressed to R.K. or S.V.S.

Reprints and permissions information is available at www.nature.com/reprints.

Publisher's note Springer Nature remains neutral with regard to jurisdictional claims in published maps and institutional affiliations. 
(c) (i) Open Access This article is licensed under a Creative Commons Attribution 4.0 International cc) License, which permits use, sharing, adaptation, distribution and reproduction in any medium or format, as long as you give appropriate credit to the original author(s) and the source, provide a link to the Creative Commons licence, and indicate if changes were made. The images or other third party material in this article are included in the article's Creative Commons licence, unless indicated otherwise in a credit line to the material. If material is not included in the article's Creative Commons licence and your intended use is not permitted by statutory regulation or exceeds the permitted use, you will need to obtain permission directly from the copyright holder. To view a copy of this licence, visit http://creativecommons.org/licenses/by/4.0/.

(C) The Author(s) 2021 